\title{
Electromechanical characteristics of piezoelectric converters with freely defined boundary conditions and geometry
}

\author{
G. Mieczkowski \\ Biatystok University of Technology, ul. Wiejska 45C, 15-351 Białystok, Poland, E-mail: g.mieczkowski@pb.edu.pl

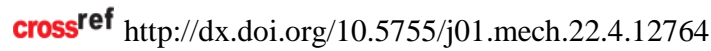

\section{Introduction}

Piezoelectric converters are used to measure and/or regulate a wide range of physical parameters such as force [1], strain [2], pressure [3], acceleration [4]. These are also used as resonators [5] or even diaphragm drives [6]. Their working principle is based on conversion of electric energy to the mechanical, or vice versa [7]. Dependency between strain and electric field is defined by constitutive equation $[8,9]$.

Practically, in the majority of piezoelectric converters, the measure/control unit is of a layer design, what causes some issues in determining their durability, and in case of bent piezoelectric converters, finding relation between bending and electric field. Both the former and the latter aspect were dealt with by many scientists.

When designing this piezoelectric converter, specific application-adequate simultaneous equations should be defined and solved. These equations bound together geometrical properties, materials properties and physical parameters, such as force, deflection and electric field. Solving such simultaneous equations is very difficult. Materials and geometrical heterogeneity of the converter's global structure and anisotropy of piezoelectric materials forces the use of some reductions. Smits et al. [10, 11], by using energetic methods, formed and solved constitutive equations for a converter made of two layers of even length (piezoelectric bimorph). Then Wang and Cross [12] extended and solved the issue of a three-layer converter, while Xiang, and Shi [13] - a multi-layer one. However in works [14, 15], results of geometrical properties optimization results for this type of converters were given.

It is a very rare case that in literature there can be found particular analytical application solutions, for some groups of piezoelectric converters, in the construction of which individual components (layers) are of different length, and boundary conditions - mounting and external load - are different as compared with solution proposed by Smits. For such cases, in order to determine electromechanical behaviour of the converter, most frequently the FEMbased analyses are carried out [16]. Preparing this type of analyses is sometimes very work-consuming however, and the solution may be subject to high error.

Therefore, the main purpose of the presented work was to develop a simple analytical method for determining deflection, in the function of mechanical and electric loads, bending piezoelectric converters of various constructions e.g. shown on Fig. 1.

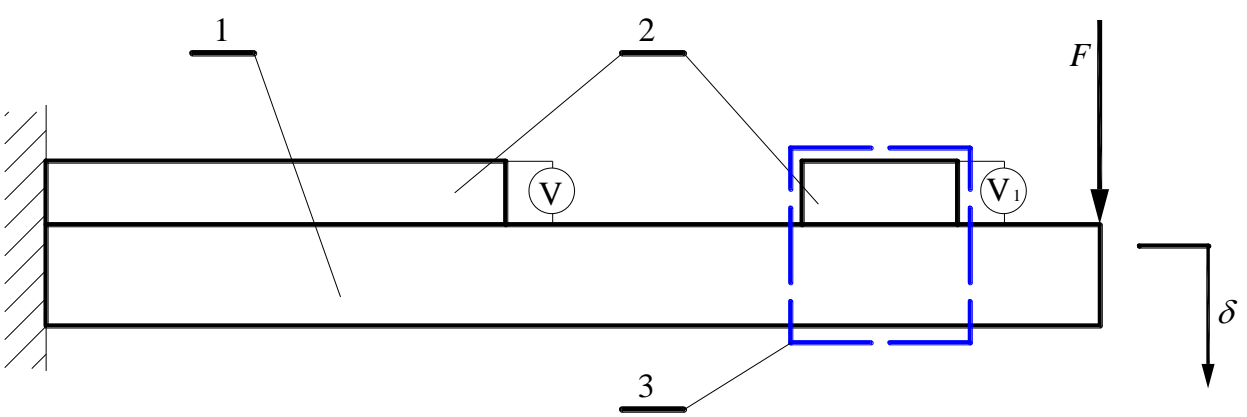

Fig. 1 Two-layer piezoelectric converter, 1-beam; 2-piezoelectric elements; 3-piezoelectric bimorph segment PBS

The proposed method involves implementation to a homogeneous beam modules referred to hereinafter "piezoelectric bimorph segment". This allows for including, within analytical description of the beam deflection, a local change in stiffness and strain caused by transverse piezoelectric effect.

In order to verify the correctness of the developed method, it is required to compare the obtained results with experimental data, or results obtained using other methods. Therefore the obtained particular solutions for the bimorph converter were compared with the literature-derived solution. For converters of diversified material and geometric structure the FEM simulations were prepared and these were compared with the obtained analytical results.

\section{Analytical simulations}

\subsection{Basic assumptions}

Converters as analysed in this work were treated as a homogeneous (one-layer) beam with locally implemented piezoelectric bimorph segments PBS (Fig. 1). The PBS comprises two components - piezoelectric and non-piezoelectric element. The non-piezoelectric layer thickness is the same as the beam thickness. The beam and the PBS both have the same width. In order to reduce the mathematical model, the following assumptions were made:

I) bending of the element takes place according to 
the Euler's hypothesis, and radii of curvatures of the deflected components are identical;

II) in the component connection plane there is no intermediate layer, and no sliding occurs,

III) in the piezoelectric layer transverse piezoelectric effect 1-3 takes place, causing clear bending.
2.2. General solution for piezoelectric converter with implemented PBS segment

The task is to consider a section of piezoelectric converter (Fig. 2) subjected to mechanical bending moment $M(x)$ and electric moment $M \mathrm{e}$ (following the occurrence of the piezoelectric effect).

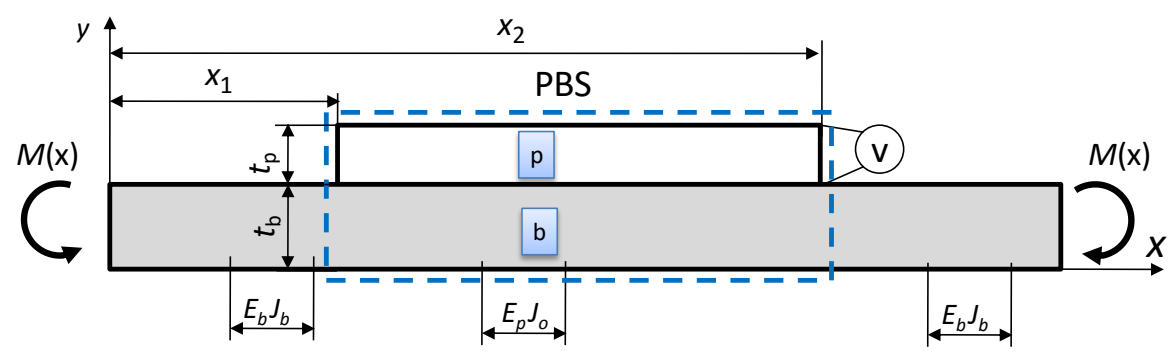

Fig. 2 Section of piezoelectric converter

In the analysed element subjected to bending, it is possible to determine three characteristic ranges, related ta change in load and stiffness. Within the $x_{1}<x<x_{2}$ range there is piezoelectric bimorph segment PBS (generating $M e$ ) with flexural stiffness $E_{p} J_{o}$. The other two ranges are homogeneous beam with stiffness $E_{b} J_{b}$. When there are several characteristic ranges on the beam, it is convenient to apply to the equation generalised functions that are determined with ranges, e.g. Heaviside function. Thus, including the PBS presence in the beam, the deflection line can be described using the following dependence:

$$
\frac{\partial^{2} y}{\partial x^{2}}=M(x) / E_{b} J_{b}+\operatorname{Me\gamma }\left(H\left[x-x_{1}\right]-H\left[x-x_{2}\right]\right),
$$

where

$$
\gamma=\frac{E_{b} J_{b}(M e+M(x))-E_{p} J_{o} M(x)}{E_{b} J_{b} E_{p} J_{o} M e} \text { - factor }
$$

including change in stiffness with applied formal notation of the Heaviside function; $H\left[x-x_{i}\right]$ - Heaviside function; $E_{p}, E_{b}$ - Young's modules of piezoelectric and non-piezoelectric element; $J_{b}, J_{o}$ - moments of inertia (described in Chapter 2.3).

As determining the mechanical moment $M(x)$ in general does not pose any problems, determining electrical load $M e$, generated by the PBS, is very burdensome and requires solving the two-dimension issue of the PBS bending.

\subsection{Piezoelectric bimorph segment PBS}

The task is to consider the bimorph segment PBS (Fig. 3), with constant width $b$, consisting of non-piezoelectric 1 and piezoelectric element 2 .

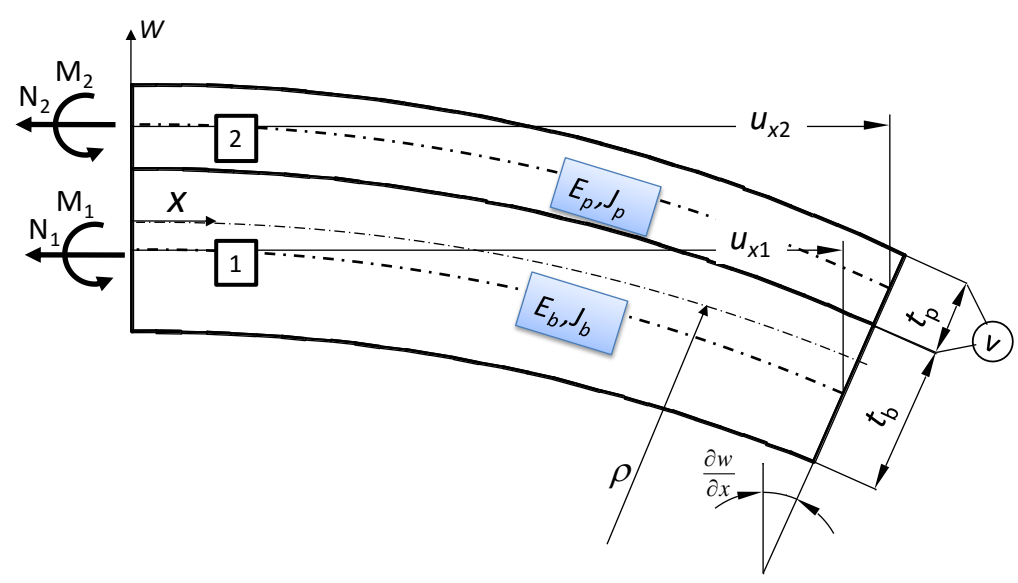

Fig. 3 Distribution of forces and conditions for strain of piezoelectric bimorph segment PBS

The bimorph is not subjected to any mechanical load, and longitudinal forces $N_{\mathrm{i}}$ and bending moments $M_{\mathrm{i}}$ occurring on individual layers are result of the applied voltage $V$. Basing on equilibrium equation of forces condition the following can be noted:

$$
N_{1}=N_{2}=N
$$

The sum of moments in relation to interface must be zero, therefore:

$$
M_{1}+M_{2}-N\left(\frac{t_{p}}{2}+\frac{t_{b}}{2}\right)=0
$$

According to the adopted Euler's hypothesis, bending moments can be notified as follows: 


$$
\left.\begin{array}{l}
M_{1}=\frac{E_{b} J_{b}}{\rho} ; \\
M_{2}=\frac{E_{p} J_{p}}{\rho} .
\end{array}\right\}
$$

Substituting dependences (4) to (3) and providing simple transformations resulted in the following:

$$
\frac{1}{\rho}=\frac{N\left(t_{p}+t_{b}\right)}{2\left(E_{b} J_{b}+E_{p} J_{p}\right)} .
$$

Including the relation between radius of curvature $\rho$ and deflection $w(x)$ :

$$
\frac{1}{\rho}=\frac{\partial^{2} w}{\partial x^{2}}
$$

Differential equation for converter bending can be notified as follows:

$$
\frac{\partial^{2} w}{\partial x^{2}}=\frac{N\left(t_{p}+t_{b}\right)}{2\left(E_{b} J_{b}+E_{p} J_{p}\right)} .
$$

Constitutive equations for lower and upper converter layers, including the piezoelectric effect in layer 2 gave the following:

$$
\left.\begin{array}{l}
\frac{\partial u_{x 1}}{\partial x}=\frac{N}{E_{b} A_{b}} ; \\
\frac{\partial u_{x 2}}{\partial x}=\frac{N}{E_{p} A_{p}}-d_{31}\left(\frac{-V}{t_{p}}\right),
\end{array}\right\}
$$

where $A_{b}, A_{p}$ - layers cross sectional areas; $d_{31}$ - piezoelectric constant.

Following relocation continuity condition (Fig. 3) it was found that:

$$
u_{x 2}-u_{x 1}-\frac{\partial w}{\partial x}\left(\frac{t_{p}}{2}+\frac{t_{b}}{2}\right)=0 .
$$

Solving differential Eqs. (7) and (8), with the following boundary conditions:

$$
\left.\begin{array}{l}
\frac{\partial w}{\partial x}(0)=0 ; w(0)=0 ; \\
u_{x 1}(0)=0 ; u_{x 2}(0)=0
\end{array}\right\}
$$

and applying dependence (9) longitudinal force $N$ can be determined:

$$
N=-\frac{b E_{b} E_{p} V d_{31} t_{b}\left(E_{b} t_{b}^{3}+E_{p} t_{p}^{3}\right)}{\beta},
$$

where $\beta=E_{\mathrm{b}}{ }^{2} t_{\mathrm{b}}^{4}+E_{\mathrm{p}}{ }^{2} t_{\mathrm{p}}^{4}+2 E_{\mathrm{b}} E_{\mathrm{p}} t_{\mathrm{b}} t_{\mathrm{p}}\left(2 t_{\mathrm{b}}^{2}+3 t_{\mathrm{b}} t_{\mathrm{p}}+t_{\mathrm{p}}^{2}\right)$.

Differential equation for bending PBS, in the $M e$, moment function, can be notified as follows:

$$
\frac{\partial^{2} w}{\partial x^{2}}=\frac{-M e}{E_{p} J_{o}}
$$

On basis of comparing dependences (7) and (12) it is possible to determine bending moment $M e$ which is the result of the piezoelectric effect:

$$
M e=-\frac{E_{p} J_{o}\left(N t_{b}+N t_{p}\right)}{2\left(E_{b} J_{b}+E_{p} J_{p}\right)},
$$

where moments of inertia for the individual layers are, respectively:

$$
\left.\begin{array}{l}
J_{b}=\frac{b t_{b}^{3}}{12} \\
J_{p}=\frac{b t_{p}^{3}}{12} .
\end{array}\right\}
$$

The averaging value of the moment of inertia $J_{o}$ can be calculated using the method of transformation of the cross sectional area [17]. Two materials of different stiffness modules and the same width $b$ (Fig. 4, a) are replaced with one material of the section composed of two parts of different widths (Fig. 4, b).

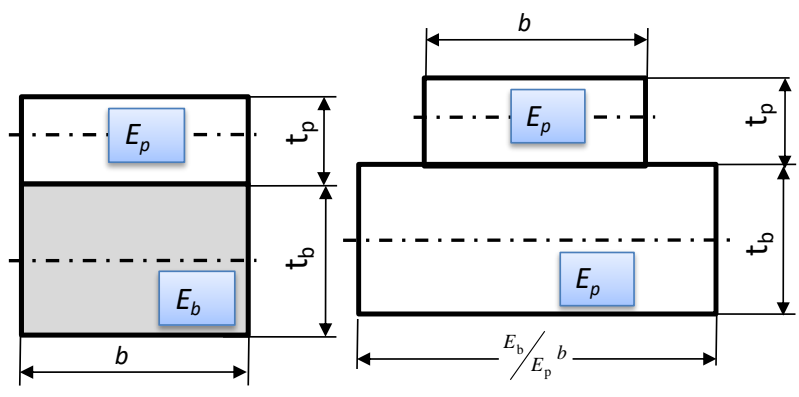

a

b

Fig. 4 Original (a) and transformed (b) section in piezoelectric bimorph segment PBS

The sought moment of inertia, calculated in relation to neutral layer, is:

$$
J_{o}=\frac{b \beta}{12 E_{p}\left(E_{b} t_{b}+E_{p} t_{p}\right)} .
$$

Substituting the formula (13) with dependences (11), (14) and (15) results in electric bending moment value in the function of the applied voltage V:

$$
M e=\frac{b E_{b} E_{p} V d_{31} t_{b}\left(t_{b}+t_{p}\right)}{2\left(E_{b} t_{b}+E_{p} t_{p}\right)} .
$$

\subsection{Particular solutions}

This part of the work is concerned with application use of the proposed method, based on implementing PBS segments into single-layer beam to determine analytical dependences describing converters' bending of fixed geometry and known boundary conditions. Solutions for converters of 
different support and position conditions and PBS quantity shall be presented.

2.4.1. Cantilever converter subjected to concentrated force $F$ of single PBS segment

In the converter as shown on Fig. 5, the left side is fix-mounted, and the right can move freely. The load results from external force $F$ and electric moment $M e$ generated by the applied voltage $V$. Based on conditions for equilibrium of forces and moments values for the reaction in mounting were established, and are as follows: $R_{y}=F ; R_{x}=0 ; M_{F}=F L$.

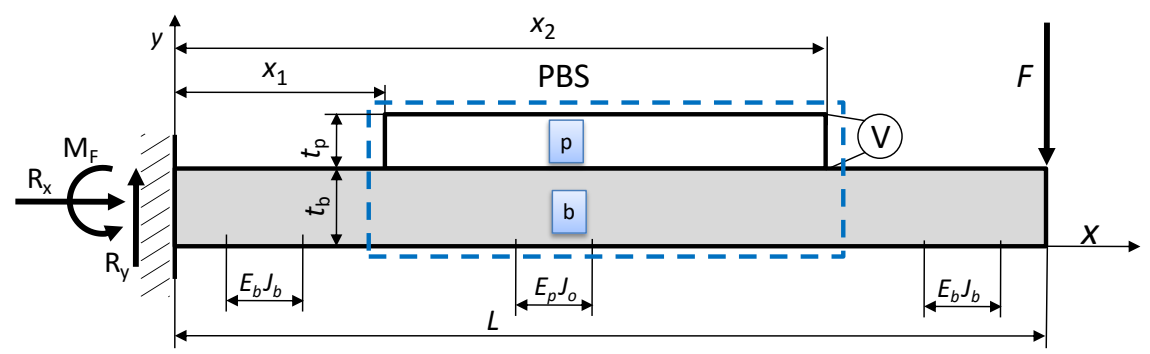

Fig. 5 Cantilever converter of single PBS segment

form:

Mechanical moment $M(x)$ takes the following

$$
M(x)=-M_{F}+R_{y} x=-F L+F x .
$$

Substituting dependences (16) and (17) to the general solution, described with formula (1), upon double integration, gives a dependence describing function of bending of the analysed converter (18):

$$
y(x)=A_{1} V+B_{1} F
$$

where

$$
\begin{aligned}
& A_{1}=\frac{3 d_{31} E_{b} E_{p} t_{b}\left(t_{b}+t_{p}\right)}{\beta}\left(\begin{array}{l}
\left(x-x_{1}\right)^{2} H\left[x-x_{1}\right]- \\
-\left(x_{2}-x\right)^{2} H\left[x-x_{2}\right]
\end{array}\right) ; \\
& B_{1}=\frac{2}{b \beta E_{b} t_{b}^{3}}\left(\begin{array}{l}
\alpha\left(\begin{array}{l}
H\left[x-x_{1}\right]\left(3 L-x-2 x_{1}\right)\left(x-x_{1}\right)^{2}- \\
-H\left[x-x_{2}\right]\left(3 L-x-2 x_{2}\right)\left(x-x_{2}\right)^{2}
\end{array}\right)- \\
-\beta x^{2}(3 L-x)
\end{array}\right) ;
\end{aligned}
$$$$
\alpha=E_{p} t_{p}\left(E_{p} t_{p}^{3}+E_{b} t_{b}\left(3 t_{b}^{2}+6 t_{b} t_{p}+4 t_{p}^{2}\right)\right) .
$$

Integration constants are determined on basis of the following boundary conditions:

$$
\frac{\partial y}{\partial x}(0)=0 ; y(0)=0
$$

Assuming in the obtained solution $x_{1}=0$ and $x_{2}=L$ gives a solution which is identical as provided in work [10].

2.4.2. Cantilever converter subjected to concentrated force $F$ of two PBS segments

For the converter shown on Fig. 6 conditions of mounting and mechanical load are identical as the described in Chapter 2.4.1. Electrical load is generated by two PBS segments powered by voltage $V_{1}$ and $V_{2}$.

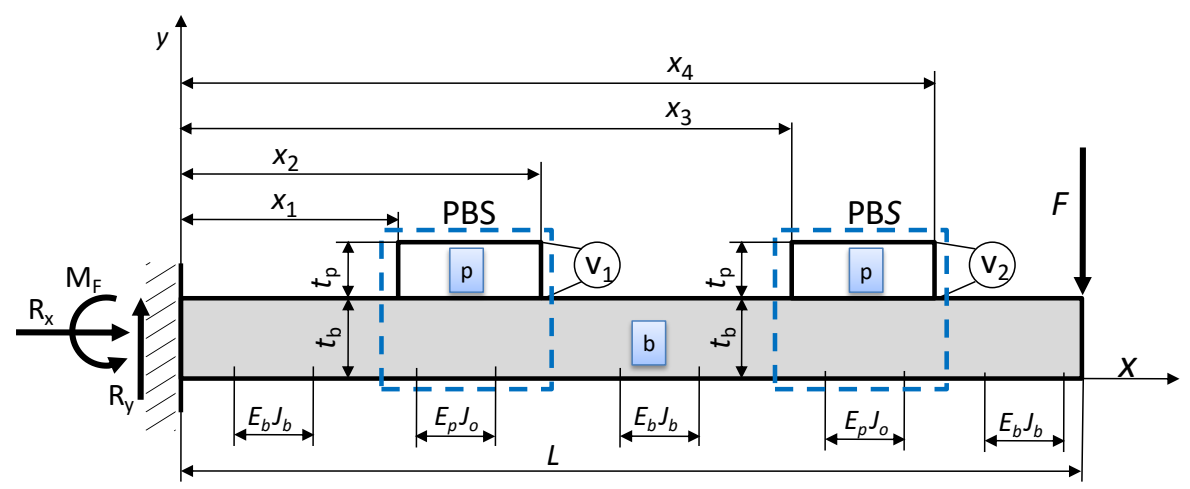

Fig. 6 Cantilever converter of two PBS segments

Differential equation for bending is as follows:

$$
\begin{aligned}
& \frac{\partial^{2} y}{\partial x^{2}}=M(x) / E_{\mathrm{b}} J_{\mathrm{b}}+M e_{1} \gamma\left(H\left[x-x_{1}\right]-H\left[x-x_{2}\right]\right)+ \\
& +M e_{2} \gamma\left(H\left[x-x_{3}\right]-H\left[x-x_{4}\right]\right) .
\end{aligned}
$$

In the above dependence mechanical moment $M(x)$ is described by Eq. (17), while electrical moments are:

$$
M e_{i}=\frac{b E_{b} E_{p} V_{i} d_{31} t_{b}\left(t_{b}+t_{p}\right)}{2\left(E_{b} t_{b}+E_{p} t_{p}\right)}, i=1,2 .
$$


Solving differential Eq. (20), assuming boundary conditions (19) gives function describing the bending of the analysed converter (22):

$$
y(x)=A_{1} V_{1}+A_{2} V_{2}+B_{2} F,
$$

where

$$
\begin{gathered}
A_{2}=\frac{3 d_{31} E_{b} E_{p} t_{b}\left(t_{b}+t_{p}\right)}{\beta}\left(\left(x-x_{3}\right)^{2} H\left[x-x_{3}\right]-\left(x_{4}-x\right)^{2} H\left[x-x_{4}\right]\right) ; \\
B_{2}=\frac{2}{b \beta E_{b} t_{b}^{3}}\left(\begin{array}{l}
\alpha\left(\begin{array}{l}
H\left[x_{1}\right]\left(3 L-x-2 x_{1}\right)\left(x-x_{1}\right)^{2}-H\left[x-x_{2}\right]\left(3 L-x-2 x_{2}\right)\left(x-x_{2}\right)^{2}+ \\
+H\left[x-x_{3}\right]\left(3 L-x-2 x_{3}\right)\left(x-x_{3}\right)^{2}-H\left[x-x_{4}\right]\left(3 L-x-2 x_{4}\right)\left(x-x_{4}\right)^{2}
\end{array}\right)- \\
-\beta x^{2}(3 L-x)
\end{array}\right) .
\end{gathered}
$$

2.4.3. Converter resting on two supports subjected to concentrated force $F$

In the converter shown on Fig. 7, the left and right

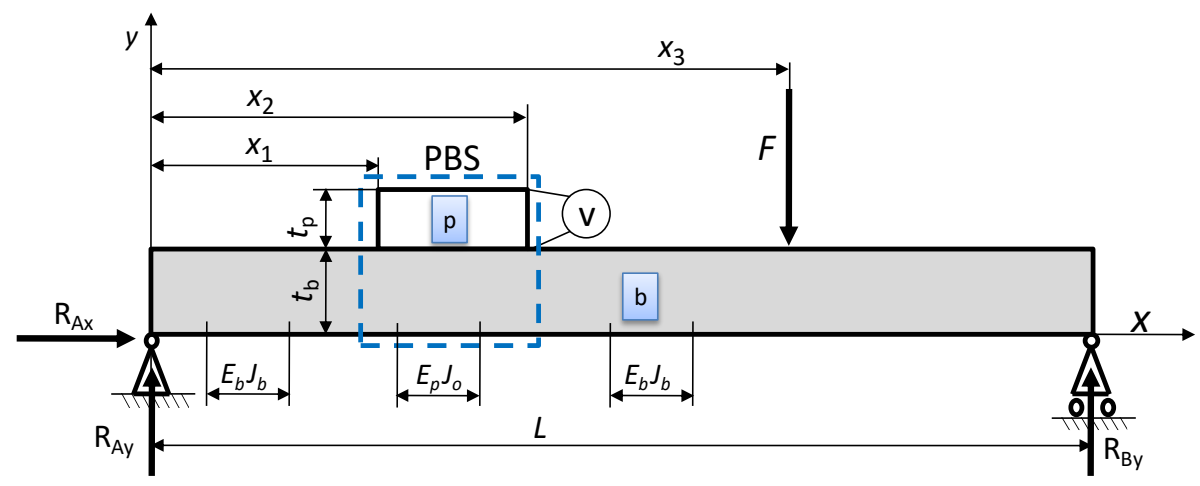

Fig. 7 Converter resting on two supports of single PBS segment

Based on conditions for equilibrium of forces and moments values for reaction in supports were determined, and are as follows: $R_{A y}=F-\frac{F x_{3}}{L} ; R_{B y}=\frac{F x_{3}}{L} ; R_{A x}=0$ and mechanical moment $M(x)$ takes the following form:

$$
M(x)=R_{A y} x-F\left(x-x_{3}\right) H\left[x-x_{3}\right] .
$$

sides are mounted resting on two supports. Alike the previously presented converters, the load derives from external force $F$ and electric moment $M e$.

$$
\begin{aligned}
& C=\frac{3 d_{31} E_{b} E_{p} t_{b}\left(t_{b}+t_{p}\right)}{\beta L}\left(L\left(x-x_{1}\right)^{2} H\left[x-x_{1}\right]-L\left(x-x_{2}\right)^{2} H\left[x-x_{2}\right]+x\left(x_{1}-x_{2}\right)\left(2 L-x_{1}-x_{2}\right)\right) ;
\end{aligned}
$$

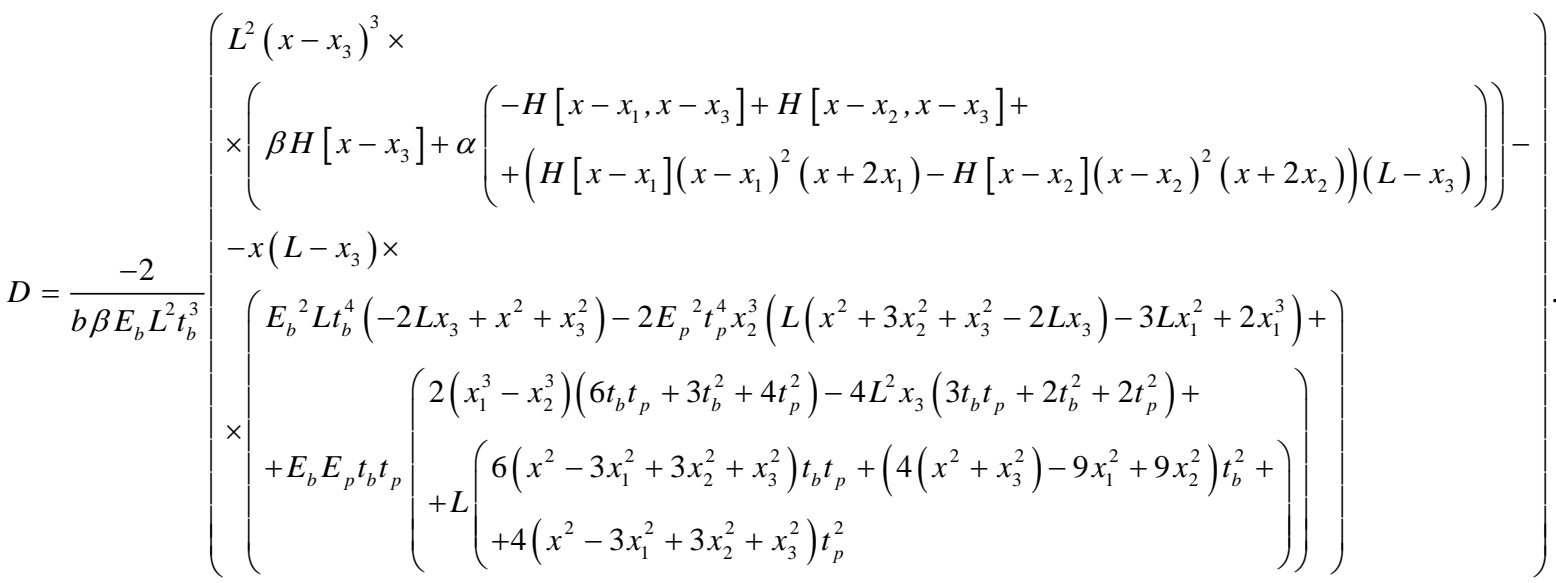

Integration constants are determined on basis of the following boundary conditions:

$$
y(0)=0, y(L)=0 .
$$




\section{Numerical calculations}

In order to verify the obtained particular solutions, it was necessary to perform numerical analyses. These tests were aimed at determining bending line of the converters for arbitrarily assumed material constants and geometry. Tested converters, shown on Figs. 5-7, were modelled using the FEM, with use of the ANSYS application [18]. Plane components were described using grid of quadrangular, eight-node finite elements, with increased concentration at critical points, such as sharp corners, mounting points and places at which mechanical load was applied. For the piezoelectric component the PLANE223 type elements were applied, and non-piezoelectric material was meshed with the PLANE183 elements. The size of finite elements was $t_{\mathrm{p}} / 4$. The plane issue was solved for two variants of stresses and strains: plane stress and plane strain conditions.

In calculations the following geometrical and material data were assumed:

- Young's modulus: $E_{p}=2.0 \times 10^{9}, E_{b}=4.0 \times 10^{9} \mathrm{~N} / \mathrm{m}^{2}$;

- Poisson's ratio: $v_{p}=0.29, v_{b}=0.33$;

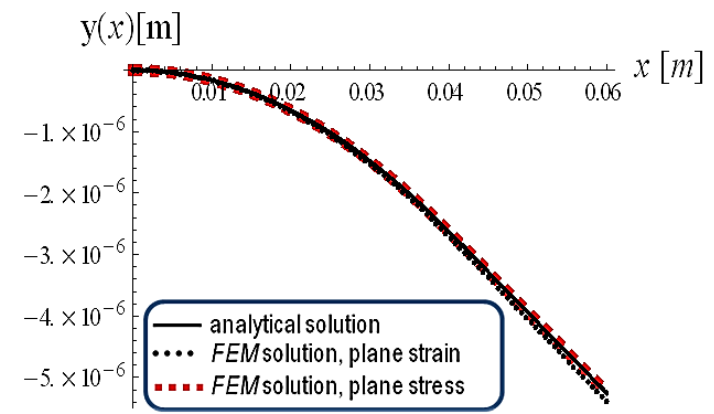

- Shear modulus: $G_{\mathrm{p}}=0.775 \times 10^{9} \mathrm{~N} / \mathrm{m}^{2}$;

- Piezoelectric strain coefficients: $d_{31}=2.2 \times 10^{-11} \mathrm{C} / \mathrm{N}$; $d_{32}=0.3 \times 10^{-11} \mathrm{C} / \mathrm{N}, d_{33}=-3.0 \times 10^{-11} \mathrm{C} / \mathrm{N}$;

- $\quad$ Relative permittivity at constant stress: $\left(\varepsilon_{33}\right)^{T}=12$;

- $\quad$ Beam length $L=60 \mathrm{~mm}$;

- Layers thickness: $t_{\mathrm{p}}=0.5 ; t_{b}=1 \mathrm{~mm}$;

- Values of applied load were:

- electrode voltage $V=-100$ Volts;

- force $F=100 \mathrm{~N}$.

Coordinates $x_{i}$ (Figs. $5 \div 7$ ), defining the PBS position and force $F$ application point were given in Chapter 4.

\section{Results of tests}

This part of work shall present graphs of bending of converters for which the resulting special solutions were given in Chapter 2.4. Results obtained from analytical solutions were compared with the FEM solutions. In analytical equations, materials and geometrical data identical as the data given in Chapter 3 were applied.

Comparison of the analytical solution (18) with the FEM - for cantilever converter of single PBS segment (Fig. 5) - were provided on Figs. 8 and 9.

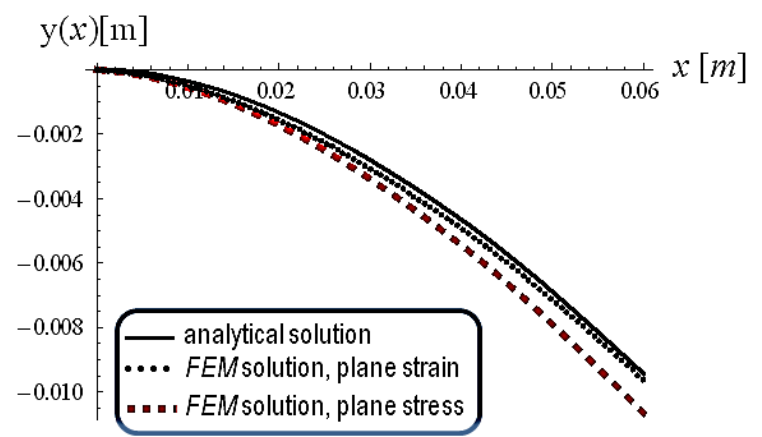

b

Fig. 8 Deflection of cantilever converter of single PBS segment for $x_{1}=0, x_{2}=2 / 3 L$ : a - subjected only to electrical voltage, $V=100 \mathrm{~V}, F=0 ; \mathrm{b}$ - subjected only to force, $V=0, F=100 \mathrm{~N}$

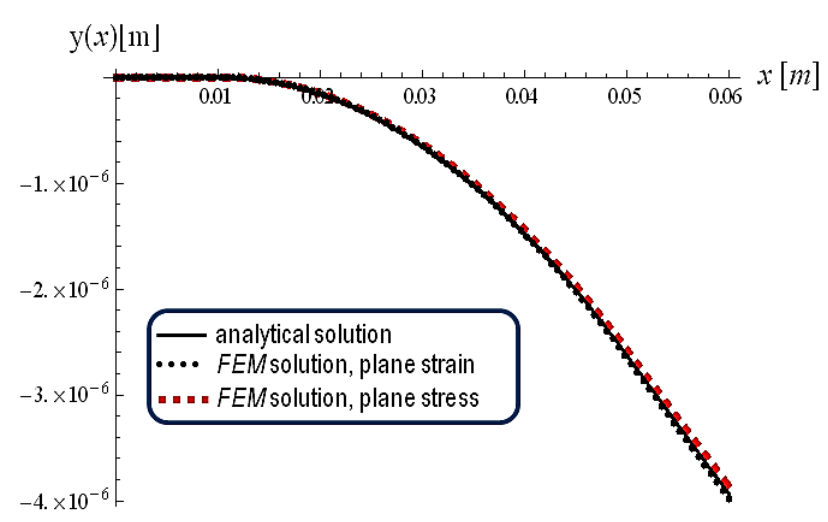

a

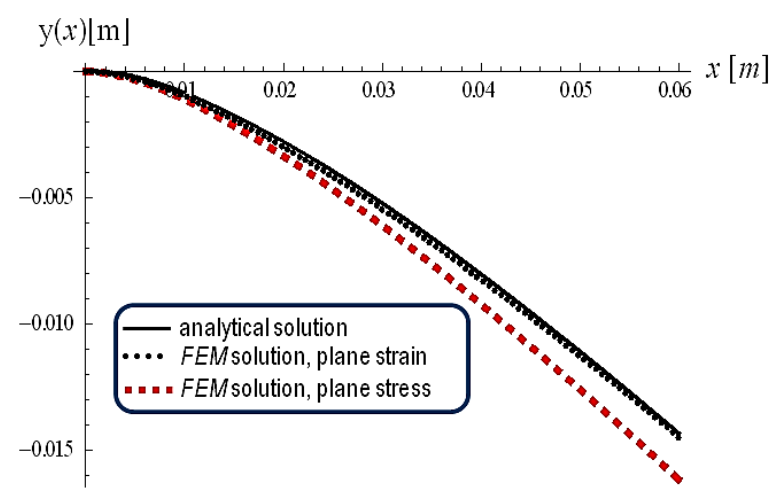

$\mathrm{b}$

Fig. 9 Deflection of cantilever converter of single PBS segment for $x_{1}=1 / 6 L, x_{2}=5 / 6 L$ : a - subjected only to electrical voltage, $V=100 \mathrm{~V}, F=0 ; \mathrm{b}$ - subjected only to force, $V=0, F=100 \mathrm{~N}$

Fig. 10 shows strain of cantilever converter of two PBS segments (Fig. 6), analytical solution for which is described as dependence (22).

Fig. 11 shows strain of converter resting on two supports of single PBS segment (Fig. 7), analytical solution for which is described as formula (24).

Basing on the obtained results, it was found that in case of cantilever converters better compatibility of analytical and numerical solutions was acquired when applied in the FEM modelling of the plane strain conditions, and for 
converter resting on two supports - plane stress.

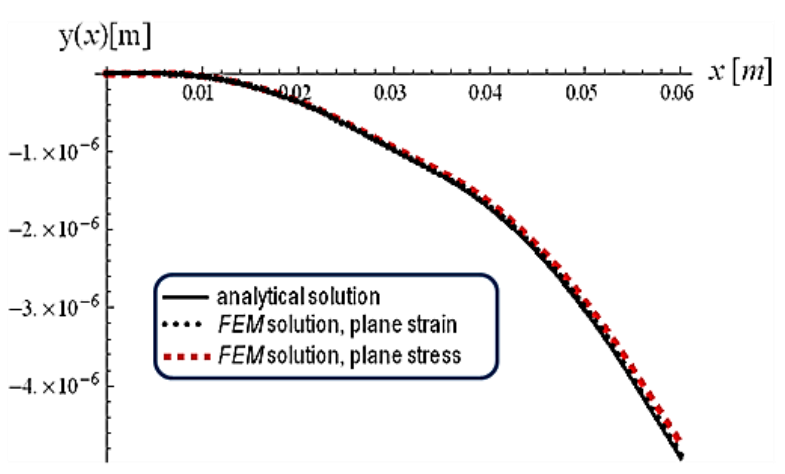

a

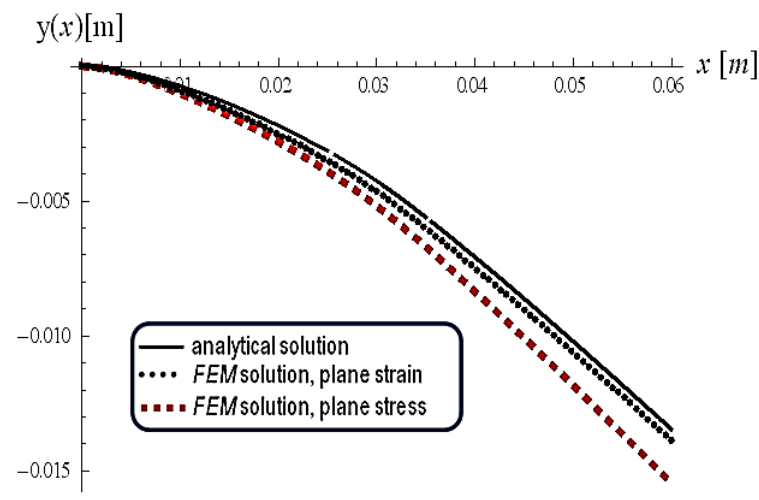

b

Fig. 10 Deflection of cantilever converter of two PBS segments for $x_{1}=1 / 12 L, x_{2}=5 / 12 L, x_{3}=7 / 12 L, x_{4}=11 / 12 L$ : a - subjected only to electrical voltage, $V_{1}=100 \mathrm{~V}, V_{2}=200 \mathrm{~V}, F=0 ; \mathrm{b}$ - subjected only to force, $V_{1}=V_{2}=0$, $F=100 \mathrm{~N}$

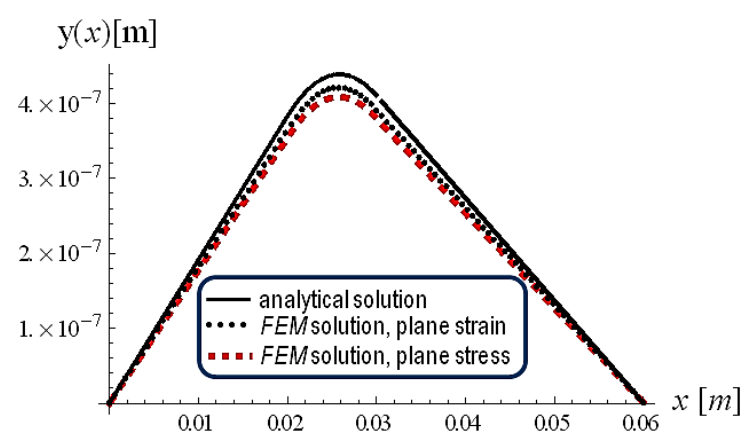

a

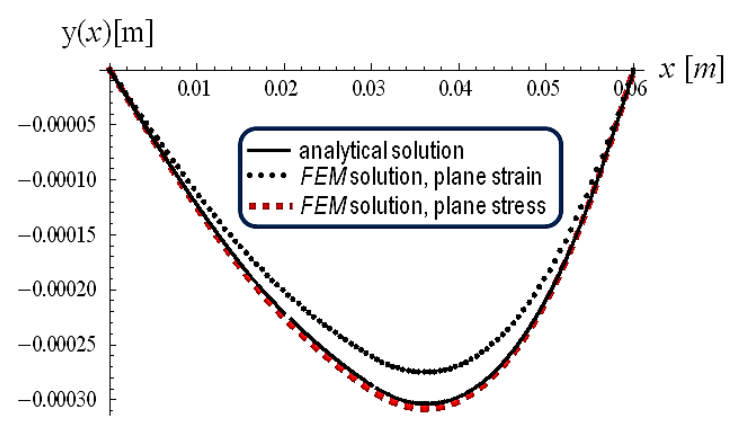

b

Fig. 11 Deflection of cantilever converter of single PBS segment for $x_{1}=1 / 3 L, x_{2}=1 / 2 L, x_{1}=1 / 3 L, x_{3}=11 / 12 L$ : a - subjected only to electrical voltage, $V=100 \mathrm{~V}, F=0 ; \mathrm{b}$ - subjected only to force, $V=0 \mathrm{~V}, F=100 \mathrm{~N}$

Generally the difference between analytical and numerical solutions was approx. $1 \%$ for electrical load, and $2 \%$ for mechanical load. Departure from the rule was the case of converter resting on two supports, for which the error at mechanical loading was approx. 9\% when applying plane strain conditions (Fig. 11b) for the FEM. It is probably caused by the way of modelling of supports for $2 \mathrm{D}$ beam. In present paper the supports were modelled as special boundary conditions. Vertical (or vertical and horizontal) displacement was blocked for only one node. For the other nodes (on left and right edges of beam) the same angle of rotation about the $z$ axis was applied as a constraint. Such change in a local stiffness probably generated errors in the numerical solution.

\section{Summary and conclusions}

The work dealt with the issue of bending, two-layer piezoelectric converters subjected to electric field and mechanical load. A general solution has been developed, based on implementation of piezoelectric segments PBS to a bending beam. Working mechanism and conditions for strain of PBS segment were determined

Basing on the general solution, for arbitrarily selected three different types of converters, special solutions were developed (for cantilever converter of single and two PBS segments and converter resting on two supports of single PBS segment). The resulting analytical solutions were compared with literature data, and the developed FEM solution.

On basis of the performed analytical and numerical tests it was found that:

- the developed method involving implementation of PBS segments into bending beams allows for obtaining solutions for piezoelectric converters:

a) of either type of mounting and external load;

b) of diverse lengths and heights of piezoelectric and non-piezoelectric layers;

c) with any numbers of piezoelectric components;

- the obtained particular solution for cantilever converter of single PBS segment, assuming the layers are of the same length, conforms to the solution provided in [10];

- other analytical particular solutions conform to the results obtained from the FEM (for electrical load maximum difference is approx. $1 \%$, and for mechanical load - approx. $2 \%)$.

\section{References}

1. Ştefănescu, D.M. 2011. Handbook of Force Transducers. Berlin: Springer-Verlag, 612 p. http://dx.doi.org/10.1007/978-3-642-18296-9.

2. Gama, A.L.; Morikawa, S.R.K. 2008. Monitoring fatigue crack growth in compact tension specimens using piezoelectric sensors, Experimental Mechanics 48(2): 247-252. 
http://dx.doi.org/10.1007/s11340-007-9086-0.

3. Caliano, G.; Lamberti, N.; Iula, A.; Pappalardo, M. 1995. A piezoelectric bimorph static pressure sensor, Sensors and Actuators A 46(1-3): 176-178. http://dx.doi.org/10.1016/0924-4247(94)00885-L.

4. Levinzon, F. 2015. Piezoelectric Accelerometers with Integral Electronics, Springer, 169 p. http://dx.doi.org/10.1007/978-3-319-08078-9_1.

5. DeVoe, D.L. 2001. Piezoelectric thin film micromechanical beam resonators, Sensors and Actuators A88(3): 263-272. http://dx.doi.org/10.1016/S0924-4247(00)00518-5.

6. Tzou, H.S. 1999. Piezoelectric Shells: Distributed Sensing and Control of Continua, Kluwer Academic Publishers, Dordrecht, $472 \mathrm{p}$. http://dx.doi.org/10.1007/978-94-011-1783-8.

7. Busch-Vishniac, I.J. 1999. Electromechanical Sensors and Actuators, Springer-Verlag, 343 p. http://dx.doi.org/10.1007/978-1-4612-1434-2.

8. Curie, P.J.; Curie J. 1880. Crystal Physics-Development by Pressure of Polar Electricity in Hemihedral Crystals with Inclined Faces, Acad. Sci.(Paris) C. R. Hebd. Seances 91, 294p. (in French)

9. Berlincourt, D.A.; Curran, D.R.; Jaffe, H.; Mason, W.P. 1964. Physical Acoustics, Principles and Methods, 1, A, Academic Press, New York. http://dx.doi.org/10.1126/science.145.3639.1424-a.

10. Smits, J.G.; Choi, W. 1991. The constituent equations of piezoelectric heterogeneous bimorphs, Ultrasonics, IEEE Trans. Ultrason. Ferroelec.Freq. Contr. 38: 256270. http://dx.doi.org/10.1109/58.79611.

11.Smits, J.G.; Dalke, S.I.; Cooney T.K. 1991. The constituent equations of piezoelectric bimorphs, Sensors and Actuators A, 28: 41-61 http://dx.doi.org/10.1016/0924-4247(91)80007-C.

12. Wang, Q.; Cross, L.E. 1999. Constitutive equations of symmetrical triple-layer piezoelectric benders, IEEE Trans. Ultrason. Ferroelec.Freq. Contr. 46: 1343-1351. http://dx.doi.org/10.1109/58.808857.

13. Xiang, H.J.; Shi, Z.F. 2008. Static analysis for multilayered piezoelectric cantilevers, International Journal Of Solids And Structures 45(1): 113-128. http://dx.doi.org/10.1016/j.ijsolstr.2007.07.022.

14. Wood, R.J.; Steltz, E.; Fearing, R.S. 2005. Optimal energy density piezoelectric bending actuators, Sensors and Actuators A 119: 476-488.

http://dx.doi.org/10.1016/j.sna.2004.10.024.

15. Correia, V.M.F; MotaSoares, C.M; MotaSoares, C.A. 1999. Optimal design of composite structures with integrated piezoelectric laminae, Mechanics of Composite Materials and Structures, NATO Science Series, 361: 389-408.

http://dx.doi.org/10.1007/978-94-011-4489-6_26.

16. Rahmoune, M.; Osmont, D. 2010. Classic finite elements for simulation of piezoelectric smart structures, Mechanika,6(86): 50-57.

http://zurna-

las.mechanika.ktu.lt/files/Mech686/Rahmoune686.pdf.

17. Fertis, D.G. 1996. Advanced Mechanics of Structures, 62-71.

18. Documentation for ANSYS, Coupled-Field Analysis Guide (2010).

\section{G. Mieczkowski}

\section{ELECTROMECHANICAL CHARACTERISTICS OF PI- EZOELECTRIC CONVERTERS WITH FREELY DEFINED BOUNDARY CONDITIONS AND GEOMETRY}

S u m m a r y

This work presents test results for usable characteristics of two-layer, bending piezoelectric converters subjected to electric field and mechanical load. A general solution has been developed, based on implementation of piezoelectric segments PBS to a bent beam. Working mechanism and conditions for strain of PBS segment were determined. Basing on the general solution, for cantilever converter (of single or two PBS segments) and converter resting on two supports of one PBS segment particular solutions were developed. The resulting analytical solutions were compared with literature data, and the developed FEM solution.

Keywords: piezoelectric bender, constitutive equations, deflection, analytical solutions.

Received July 26, 2015

Accepted July 04, 2016 